general anaesthesia. ${ }^{l-3}$ On the other hand, the epidural fentanyl requirement following general anaesthesia reported by Glass et al. ${ }^{4}$ is similar to ours. This substantial discrepancy, we feel, in loading and maintenance doses of epidural opioids for effective pain relief may have been due to the difference in anaesthetic technique. Recent reports have shown that intraoperative interruption of nociceptive impulses by regional anaesthesia results in prolonged reduction in postoperative pain. ${ }^{5.6}$ In our study, respiratory depression that required natoxone developed in two patients following 3 and $5 \mathrm{mg}$ epidural morphine, respectively, and there was no relationship between loading doses and other side effects in either group of patients. Although we admit that more than enough opioids were given in some patients, we feel that the side effects and the temporal course of those side effects we observed reflect what is expected to occur with epidural opioids in orthopaedic patients following general anaesthesia when adequate pain relief is obtained.

We agree with Dr. Chrubasik et al. that the quality of pain relief may be better with epidural morphine. The mood of our patients receiving epidural morphine was better (significantly at the $48 \mathrm{th} \mathrm{hr}$ ). Those receiving epidural fentanyl were often dysphoric. We wonder whether this was related to the systemic concentrations of fentanyl. With meperidine, plasma concentrations below those that produce mild analgesia are often associated with lethargy and pyschomotor impairment. ${ }^{7}$ We concur with Dr. Chrubasik et al. that some form of combined use of epidural opioids together with intraoperative use of regional technique and perhaps other form of pain medication, e.g., antiprostaglandin, ${ }^{8}$ may provide pain relief of better quality, less side-effects, and a safer technique.

Martin J. White MD FCAnaes

Stephen W. Dumont MB FFARCS

Michael F. Heine MD

Kentaro Tsueda MD

Julia A. Schroeder MD

Department of Anesthesiology

University of Louisville

Louisville, KY 40292

REFERENCES

1 Martin R, Salbaing J, Blaise G, Tetrault JP, Tetrault L. Epidural morphine for postoperative pain relief: a doseresponse curve. Anesthesiology 1982; 56: 5423-6.

2 Chrubasik J, Wust H, Schulte-Monting J, Thon K, Zindler $M$. Relative analgesic potency of epidural fentanyl, alfentanil and morphine in treatment of postoperative pain. Anesthesiology 1988; 68: 929-33.

3 Chrubasik J, Chrubasik S, Magora F. Pharmacokinetic profiles under morphine, methadone, fentanyl and sufentanil epidural patient-controlled analgesia (ePCA). Anesthesiology 1991; 75: A753.

4 Glass PSA, Estok P, Ginsberg B, Goldberg JS, Sladen $R N$. Use of patient-contrtolled analgesia to compare the efficacy of epidural to intravenous fentanyl administration. Anesth Analg 1992; 74: 345-51.

5 Tverskoy M, Cozacor C, Ayache M, Bradley Jr EL, Kissin I. Postoperative pain after inguinal herniorrhaphy with different types of anesthesia. Anesth Analg 1990; 70: 29-35.

6 Jebeles JA, Reilly JS, Gutierrez JF, Bradley EL, Kissin I. The effect of preincisional infiltration of tonsils with bupivacaine on the pain following tonsillectomy under general anesthesia. Pain 1991; 47: 305-8.

7 Mather Le, Meffin PF. Clinical pharmacokinetics of pethidine. Clin Pharmacokinet 1978; 3: 352-69.

8 Reasbeck $P G$, Rice $M L$, Reasbeck JC. Double-blinded controlled trial of indomethacin as an adjunct to narcotic analgesia after major abdominal surgery. Lancet 1982; 2: $115-9$.

\section{LMA for failed intubation (1)}

To the Editor:

Drs. Priscu, Priscu and Soroker presented a case of failed intubation in obstetrics in which a laryngeal mask airway (LMA) was used successfully.' They concluded that the decision to use an LMA should be made "very early" after failure to intubate. However, two recent papers provide evidence to refute this claim. Rabey et al. found that LMA insertion caused a decrease in lower oesophageal sphincter (LOS) barrier pressure in anaesthetised, spontaneously breathing patients. ${ }^{2}$ In a control group, anaesthesia was maintained with face mask and Guedel airway. LOS barrier pressure increased. Ansermino and Blogg compared insertion of the LMA with and without cricoid pressure. LMA insertion was successful at the first attempt in only two of the 20 patients who had cricoid pressure applied. ${ }^{3}$ The authors suggest that in a patient at risk of regurgitation for whom ventilation can be maintained with a face mask while cricoid pressure is applied, it would be safer to continue with face mask anaesthesia than try to insert an LMA. They contend that insertion of an LMA may be an alternative to cricothyroid cannulation.

Tunstall emphasized that the original failed intubation drill was conceived to prevent death from aspiration of vomit following regurgitation. ${ }^{4}$ Drs. Priscu, Priscu and Soroker have not demonstrated due regard for oxygenation without aspiration. ${ }^{5}$

D.M. LeVy FRCA FRARCSI

Department of Anaesthesia

Sheffield University

England

\section{REFERENCES}

1 Priscu V, Priscu L, Soroker D. Laryngeal mask for failed intubation in emergency Caesarean section. Can J Anaesth 1992; 39: 893.

2 Rabey PG, Murphy PJ, Langton JA, Barker B, Rowbotham $D J$. Effect of the laryngeal mask airway on lower 
oesophageal sphincter pressure in patients during general anaesthesia. Br J Anaesth1992; 69: 346-8.

3 Ansermino JM, Blogg CE. Cricoid pressure may prevent insertion of the laryngeal mask airway. Br J Anaesth 1992; 69: 465-7.

4 Tunstall ME. Failed intubation in the parturient (Editorial). Can J Anaesth 1989; 36: 611-3.

5 Tunstall $M E$, Sheikh $A$. Failed intubation protocol: oxygenation without aspiration. Clin Anaesth 1986; 4: 171-87.

\section{LMA for failed intubation (2)}

To the Editor:

Priscu et al. ' suggested that the priorities of the various failed intubation drills in obstetric patients should be changed and the LMA used at an early stage. However, it is our view that their use of the LMA in the patient reported was inappropriate and their suggestion is unjustified.

When the patient's lungs can be ventilated adequately with a face mask, the LMA confers no advantage except that jaw support is unnecessary. We agree with the view of King and Adams ${ }^{2}$ that "should ventilation be easy after failed intubation in an obstetric patient, insertion of the LMA would at the very least be meddlesome." In addition, lower oesophageal sphincter pressure has been shown to be significantly lowered with the use of the LMA when compared with the use of the face mask and Guedel airway, ${ }^{3}$ thus increasing the risk of regurgitation. The studies to date on the effect of the LMA on the incidence of the regurgitation show conflicting results. ${ }^{3-5}$ In this context, given our present state of knowledge, we would be cautious in recommending the use of the LMA in view of the potential for regurgitation. Furthermore, since the tip of the LMA lies below the level of the cricoid cartilage when the LMA is properly positioned, application of cricoid pressure hampers correct positioning of the LMA.

When ventilation cannot be maintained after efforts at tracheal intubation have failed, the LMA could be tried. This decision should be made at an early stage, as the LMA may not always provide adequate ventilation. Whether or not cricoid pressure should be released temporarily during insertion of the LMA is controversial. ${ }^{6,7}$ Once the LMA is inserted, cricoid pressure must be applied continuously until airway reflexes return; recovery of spontaneous respiration does not guarantee return of airway reflexes.

Therefore, we believe that in patients at high risk of pulmonary aspiration, the LMA should not be used when adequate ventilation can be achieved with a face mask with effective application of cricoid pressure.

\author{
Takashi Asai MD \\ Ian Appadurai FRCA \\ Department of Anaesthetics \\ University of Wales College of Medicine \\ Heath Park, Cardiff CF4 4XN \\ United Kingdom
}

\section{REFERENCES}

1 Priscu V, Priscu L, Soroker D. Laryngeal mask for failed intubation in emergency Caesarean section. Can J Anaesth 1992; 39: 893.

2 King TA, Adams AP. Failed tracheal intubation. $\mathrm{Br} \mathrm{J}$ Anaesth 1991; 65: 400-14.

3 Rabey PG, Murphy PJ, Langton JA, Barker P, Rowbotham $D J$. Effect of the laryngeal mask airway on the lower oesophageal sphincter during anaesthesia. $\mathrm{Br} \mathrm{J}$ Anaesth 1992; 69: 346-8.

4 Barker P, Murphy P, Langton JA, Rowbotham DJ. Regurgitation of gastric contents during general anaesthesia using the laryngeal mask airway. Br J Anaesth 1991; 67: $600 \mathrm{P}$.

5 El Mikatii N, Luthra AD, Healy TEJ, Mortimer AJ. Gastric regurgitation during general anaesthesia in the supine position with the laryngeal and face mask airways. $\mathrm{Br}$ J Anaesth 1992; 69: 529-30P.

6 Ansermino JM, Blogg CE. Cricoid pressure may prevent insertion of the laryngeal mask airway. $\mathrm{Br} \mathrm{J}$ Anaesth 1992; 69: 465-7.

7 Brimacombe J. Cricoid pressure and the laryngeal mask airway. (Letter). Anaesthesia 1991; 46: 986-7.

\section{LMA for failed intubation (3)}

To the Editor:

The most interesting feature of this case was the decision to convert to the LMA after adequate ventilation had been achieved with a face mask (FM). We would like to question whether this manoeuvre is appropriate in the non-fasted patient. To change from one form of airway management which has been found to be adequate with cricoid pressure (CP) applied, to an alternative where adequate oxygenation is not guaranteed is potentially hazardous. We feel this could only be advised if continued airway patency was in doubt or the risks of aspiration was considered substantially less with the alternative technique.

The concerns regarding the use of the LMA in the non-fasted patient include the interaction between CP and 